\title{
The Radiative, Cloud, and Thermodynamic Properties of the Major Tropical Western Pacific Cloud Regimes
}

\author{
CHRISTIAN JAKOB \\ BMRC, Melbourne, Australia \\ GeORge TseLIOUdis \\ NASA GISS/Department of Applied Physics, Columbia University, New York, New York \\ TiмOTHY Hume \\ BMRC, Melbourne, Australia
}

(Manuscript received 27 April 2004, in final form 16 August 2004)

\begin{abstract}
This study investigates the radiative, cloud, and thermodynamic characteristics of the atmosphere separated into objectively defined cloud regimes in the tropical western Pacific (TWP). A cluster analysis is applied to $2 \mathrm{yr}$ of daytime-only data from the International Satellite Cloud Climatology Project (ISCCP) to identify four major cloud regimes in the TWP region. A variety of data collected at the Department of Energy's Atmospheric Radiation Measurement Program (ARM) site on Manus Island is then used to identify the main characteristics of the regimes. Those include surface and top-of-the-atmosphere radiative fluxes and cloud properties derived from a suite of ground-based active remote sensors, as well as the temperature and water vapor distribution measured from radiosondes.

The major cloud regimes identified in the TWP area are two suppressed regimes-one dominated by the occurrence of mostly shallow clouds, the other by thin cirrus-as well as two convectively active regimesone exhibiting a large coverage of optically thin cirrus clouds, the other characterized by a large coverage with optically thick clouds. All four of these TWP cloud regimes are shown to exist with varying frequency of occurrence at the ARM site at Manus. It is further shown that the detailed data available at that site can be used to characterize the radiative, cloud, and thermodynamic properties of each of the regimes, demonstrating the potential of the regime separation to facilitate the extrapolation of observations at one location to larger scales. A variety of other potential applications of the regime separation are discussed.
\end{abstract}

\section{Introduction}

Clouds play several important roles in the climate system. The most prominent of those is the modification of the radiative fluxes throughout the atmosphere and at the earth's surface. Other effects include the release and consumption of latent heat related to phase changes of water within clouds or in precipitation generated in them; the transport of heat, moisture, momentum, and trace constituents in convective clouds; and the modification of the land surface hydrology through precipitation. The processes involved in these modifications are complex and involve feedbacks that are often poorly understood. It is therefore not surprising that the representation of clouds in atmospheric models

Corresponding author address: Dr. Christian Jakob, BMRC, GPO Box 1289K, Melbourne, VIC 3001, Australia.

E-mail: c.jakob@bom.gov.au is often poor, in particular in those models whose grid size requires both the cloud dynamics and physics to be parameterized. This has led to the repeated conclusion in the climate change community that the poor understanding of cloud effects and feedbacks together with the difficulties in modeling clouds remain major stumbling blocks in our ability to estimate future climate change (e.g., Houghton et al. 2001).

One way of improving our understanding of the role clouds play is by separating the multitude of clouds observed into discernible cloud regimes and studying their individual properties. This approach has a long tradition, beginning with the classification of clouds by their visible characteristics by Luke Howard in 1802 (e.g., Stephens 2003). Today, entire research programs, such as the Global Energy and Water Cycle Experiment (GEWEX) Cloud System Study (GCSS; Browning 1993; Randall et al. 2003), concentrate their efforts into the study of separable cloud regimes, with the 
separation often made intuitively rather than objectively. Subjective cloud regime identification is straightforward in areas where cloud types are geographically separated, such as in the vast areas of the eastern subtropical oceans covered by stratocumulus clouds. In other areas, such as the midlatitude storm tracks and large areas of the Tropics, large variability prevents the use of a simple subjective classification.

Recently, a number of studies have proposed ways to objectively identify cloud regimes in those areas with the aim to better understand the link of clouds to the dynamic and thermodynamic environments they are embedded in as well as their feedbacks onto those environments (e.g., Lau and Crane 1995; Bony et al. 1997; Tselioudis et al. 2000; Webb et al. 2001; Norris and Weaver 2001; Tselioudis and Jakob 2002; Williams et al. 2003; Jakob and Tselioudis 2003, hereafter JT03). Apart from aiming at improving our understanding of cloud effects both in the current and future climate, many of these studies were also carried out to identify weaknesses in the representation of clouds in general circulation models (GCMs). Jakob (2003) argued that exposing model shortcomings as a function of cloud regime is an important step in improving GCMs by providing a crucial link between a model's climate and the model's actual cloud representation. The latter can often only be studied in detailed process studies based on individual cases and simplified versions of the GCM, such as single-column models (SCMs; Randall et al. 1996), and studies of this kind can easily become detached from the problems encountered in a model's climate.

JT03 recently applied a simple cluster analysis technique to data from the International Satellite Cloud Climatology Program (ISCCP; Rossow and Schiffer 1983, 1999) in the tropical western Pacific (TWP). They show that cloud fields can be grouped into four major cloud regimes: two convectively active and two suppressed. JT03 suggest a number of applications of the regime information they derived, ranging from understanding cloud feedbacks in terms of within-regime and regime-occurrence variations to the evaluation of regime-dependent model errors in GCMs. The applicability of the regimes identified in the TWP by JT03 for these purposes hinges on the assumption that they represent relevant cloud "modes" of the tropical atmosphere. As the quotes indicate, the term modes is used loosely here and throughout the manuscript to describe recurring cloud field configurations at the scale of an ISCCP grid box $(280 \mathrm{~km} \times 280 \mathrm{~km})$. While the results presented in JT03 are intuitively interpretable, no evidence that those regimes, as well as their relation to radiative and thermodynamic characteristics of the atmosphere, are distinct has been given. The aim of this study is to provide such evidence and to comprehensively describe the cloud, radiative, and thermodynamic characteristics of each of the four TWP cloud regimes.

Data collected by the Department of Energy's (DOE)
Atmospheric Radiation Measurement Program (ARM; Stokes and Schwartz 1994; Ackerman and Stokes 2003) at its TWP Cloud and Radiation Test Bed (CART) site located on Manus Island (Mather et al. 1998) during 1999-2000 are applied to identify the cloud regime properties. Several data sources, such as radiative fluxes at the surface and top of the atmosphere (TOA), cloud characteristics derived from ground-based active remote sensors, and thermodynamic information from radiosondes as well as remotely sensed total column water vapor are used to characterize the TWP cloud regimes identified in JT03. Apart from providing evidence that the regimes do in fact represent distinct cloud modes, the analysis will describe details on the impact of the latter on the radiation fields. It will also expose some limitations of identifying cloud regimes from satellite information, by contrasting the satellitederived cloud properties with those identified by active, ground-based remote sensors.

Section 2 will introduce the TWP cloud regimes as they were identified in JT03 and provide some background on their definition as well as their occurrence at the Manus ARM site. Section 3 contains the main findings of the study. It describes the radiative, cloud, and thermodynamic characteristics associated with each of the cloud regimes as identified using a variety of data sources available at the ARM site. Section 4 will discuss the results in the context of their potential application to a wide range of problems both in model development and data analysis, and section 5 summarizes the main findings and conclusions.

\section{The TWP cloud regimes}

The data source for the identification of the TWP cloud regimes are 3-hourly histograms of the frequency of occurrence of cloudy satellite pixels exhibiting a certain combination of cloud-top pressure (CTP) and cloud optical thickness $(\tau)$. These data are provided by the ISCCP D1 data product (Rossow and Schiffer 1999) on a $280 \mathrm{~km} \times 280 \mathrm{~km}$ grid. The histograms are generated by subsampling within each gridbox and 3-h interval satellite pixels of about $5-\mathrm{km}$ resolution every 30 $\mathrm{km}$. The data are grouped into seven CTP and six $\tau$ groups leading to frequencies of occurrence reported in 42 classes. The necessity of information from a visible channel in the retrieval of optical thickness limits the data availability to the local daytime hours.

JT03 used histograms collected for the year of 1999 in the TWP area, which is defined as $10^{\circ} \mathrm{N}-10^{\circ} \mathrm{S}, 130^{\circ}-$ $170^{\circ} \mathrm{E}$. Here the use of their method is extended to include both 1999 and 2000. The $K$-means clustering algorithm (Anderberg 1973) is applied to all histograms in the TWP area for 1999-2000 (ca. 320000 data points). By design, the $K$-means analysis assigns each data vector to a cluster, so all TWP data points contrib- 
ute to the findings of this study. Initially, the cluster analysis is performed for all cluster numbers from two through eight. As in JT03, the cloud regimes yielded in a four-cluster analysis are chosen for further study. This decision is based on studying the evolution of the cluster mean histograms as cluster number is increased. In the range from two to four clusters, each analysis yields an additional cluster mean histogram that is distinctly different from those of the previous analysis. Once the cluster number increases beyond four, the resulting new cluster mean histograms tend to be visually indistinguishable from at least one of the mean histograms of the four-cluster analysis. This is accompanied by a deterioration of the convergence in the iterative $K$-means clustering algorithm, indicating that at least two clusters of any of the analyses carried out with five or more clusters are close to each other in phase space. Since this study aims to identify the major cloud regimes in the TWP, the four-cluster analysis results constitute the optimal choice. It is worthwhile noting that studies that aim to expose more subtly different regimes or those aimed at extremes will naturally have to consider a larger number of clusters.

Once the cluster mean histograms (i.e., the cluster centroids) for the entire TWP have been derived, the presence of a cloud regime at Manus Island is identified as follows. All CTP- $\tau$ histograms for the ISCCP grid area that contains Manus Island are extracted from the ISCCP dataset. This ISCCP area is centered at $1.3^{\circ} \mathrm{S}, 146.3^{\circ} \mathrm{E}$; the location of the Manus site is $2.1^{\circ} \mathrm{S}$, $147.4^{\circ} \mathrm{E}$. Each single grid area histogram is then assigned to the TWP cloud regime for which its distance (simply defined as the Euclidian vector distance) to the TWP cluster mean histogram is at a minimum. The cluster mean histograms for both the four TWP cloud regimes and their derived representation at Manus Island are displayed in Fig. 1. Comparison with Fig. 3 of JT03 shows that there is very little difference between their results and those shown here, despite the fact that an additional year of data is used. This lends further confidence to the robustness of the cloud-regime identification.

The four cloud regimes identified in Fig. 1 are (from top to bottom) as follows:

1) a suppressed (in the deep convective sense) cloud regime dominated by low-top (CTP $>680 \mathrm{hPa}$ ) clouds of low to medium $\tau$, most likely shallow convective clouds, hereafter referred to as the suppressed shallow clouds (SSC) regime;

2) a suppressed cloud regime, dominated by high-top $(\mathrm{CTP}<440 \mathrm{hPa})$ clouds of low $\tau$, hereafter referred to as the suppressed thin cirrus (STC) regime;

3) a regime with a small coverage of what are likely deep convective clouds and a large coverage of hightop $(\mathrm{CTP}<440 \mathrm{hPa})$ medium- $\tau$ cirrus, hereafter referred to as the convectively active cirrus (CC) regime;
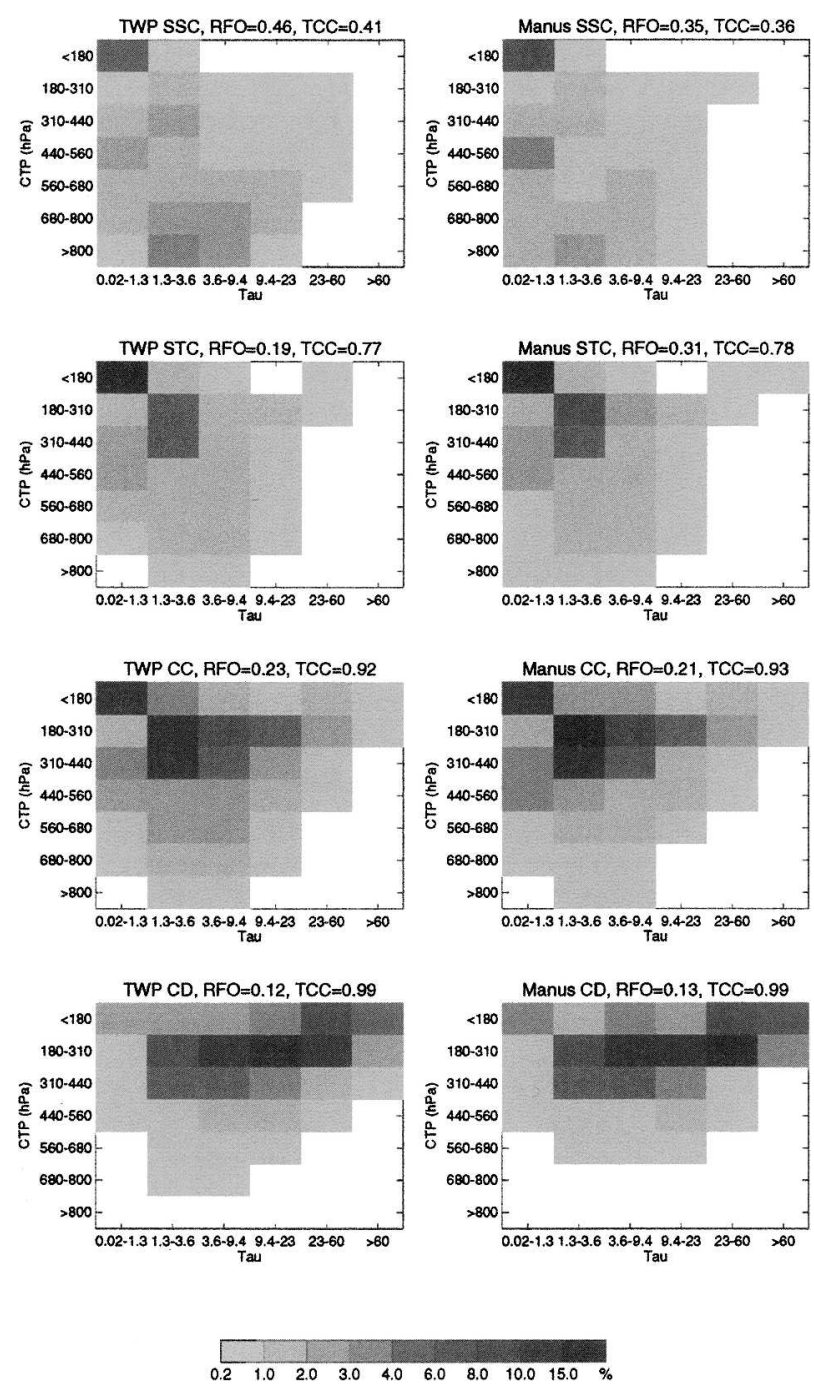

FIG. 1. Cluster mean CTP- $\tau$ histograms for $1999-2000$ as identified (left) by cluster analysis for the TWP and (right) by projection for Manus. The regimes are SSC, STC, CC, and CD. Also shown is the relative frequency of occurrence (RFO) and the total cloud cover (TCC).

4) and a regime dominated by a large coverage with high-top (CTP $<440 \mathrm{hPa}$ ) clouds of substantial optical thickness, hereafter referred to as the convectively active deep cloud (CD) regime.

Since the cloud regime information depends on the availability of the ISCCP D1 CTP $-\tau$ histograms, the presence of a particular cloud regime around Manus Island can be identified only during daytime and at 3-hourly intervals. During the time period used for this study about 3000 such histograms exist for the Manus area. Figure 2 shows an example for the time evolution of the cloud regimes over Manus in the month of May 2000. The cloud regime information indicates that the month started with a period of very strong convection (1-7 May) followed by a prolonged period of sup- 


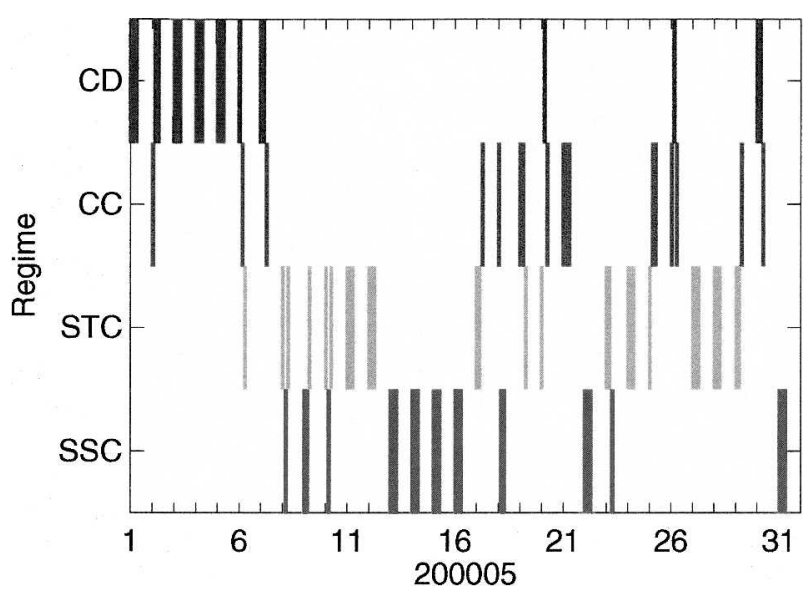

FIG. 2. Time series of the occurrence of the TWP cloud regimes around Manus Island in May 2000. An individual event is characterized by a bar of the smallest width, such as the events on 20 May. Broader bars indicate the consecutive occurrence of the same regime.

pressed conditions (8-16 May), followed by changeable conditions with short periods (1-2 days) of convection. While only meant to illustrate the assignment of a particular regime to a given time, the figure highlights an interesting direct benefit from the regime identification. Once the cloud regimes have been defined, their presence can be identified for any point of interest and a simple diagram like that shown in Fig. 2 suffices to unambiguously characterize the conditions with regards to convective activity at that point for any given time.

\section{The characteristics of the TWP cloud regimes}

With the ability of assigning a particular cloud regime to any daytime 3-h interval at Manus as described in section 2, it is now possible to use data collected at the ARM sites to comprehensively characterize each of the four TWP regimes. This is achieved by collocating in time the ARM measurements with the ISCCP information and distributing the data into four classes, each representing one of the cloud regimes. First, the radiative characteristics of the individual regimes will be described. This is followed by the description of some cloud characteristics as identified from ground-based active remote sensors. Finally, radiosonde data will be used to describe the thermodynamic state in which each of the cloud regimes tends to occur. The choice of variables investigated in this first study of the TWP cloud regime properties is driven by two main factors-the potential use of the results in future model evaluation studies and data availability. While the dataset used here is fairly comprehensive, additions from other data sources are envisaged for future work.

Since the cloud regimes are defined only over a large area $\left(280 \mathrm{~km}^{2} \times 280 \mathrm{~km}^{2}\right)$, the use of point observations to characterize the regimes is limited to making statis- tical assessments. Hence most of the following analysis will be carried out by characterizing each regime through some mean or median properties as well as by providing some information on other characteristics of the distribution of properties within each regime. This type of analysis requires long time series of the point observations and hence makes the ARM sites ideal locations to carry out such a study.

\section{a. Radiative characteristics}

The radiative characteristics of the TWP cloud regimes are determined using data collected at or around the Manus ARM CART site. Surface radiation is characterized by using hourly averages of downward longwave radiation, downward total solar radiation, and an estimate of the clear-sky value of downward solar radiation acquired using the method of Long and Ackerman [2000; the specific algorithm used here is described in Long and Gaustad (2004)]. The quantity assessed for surface solar radiation is the ratio of total to clear-sky radiation, which represents the cloud effect on the solar radiation. By choosing this quantity, possible diurnal variations in the occurrence of the individual cloud regimes are excluded. The TOA radiation is characterized using hourly retrievals of outgoing longwave radiation (OLR) and visible albedo derived from geostationary satellite information (GMS) using the approach of Minnis et al. (1995) as reported by Nordeen et al. (2001). The TOA values represent area averages for a $1^{\circ} \times 1^{\circ}$ area around Manus that were derived by averaging over nine grid boxes of the original $0.3^{\circ} \times 0.3^{\circ}$ data. Only radiation data within the hour for which the cloud regime identification exists (i.e., 3-hourly, local daytime) are used in the analysis. Periods of missing or erroneous data are removed. The surface radiation data used are available throughout 1999-2000, while for the TOA-only data for the year 2000 is available.

Figure 3 summarizes the radiative characteristics of each of the cloud regimes in the form of box whisker diagrams. Each of the panels in this figure is generated in the following way. The radiation data are first grouped by cloud regime. Then for each parameter and each regime, a box whisker diagram is constructed representing the frequency distribution of the values within each regime. The horizontal line in each box marks the distribution median, the box represents all values between the $25 \%$ and $75 \%$ quartiles, and the whiskers are drawn to the $5 \%$ and $95 \%$ percentiles. The four panels show the results for TOA OLR and albedo (top) and surface downward longwave as well as the normalized downward solar radiation (bottom).

The median values of OLR vary strongly from $280 \mathrm{~W}$ $\mathrm{m}^{-2}$ for the SSC regime to just above $160 \mathrm{~W} \mathrm{~m}^{-2}$ for the $\mathrm{CD}$ regime. The STC and CC regimes show values between the two other regimes of 250 and $210 \mathrm{~W} \mathrm{~m}^{-2}$, respectively. There is some overlap in the OLR values between the regimes (i.e., the boxes overlap), but the signal of a reduction in OLR when stepping through the 

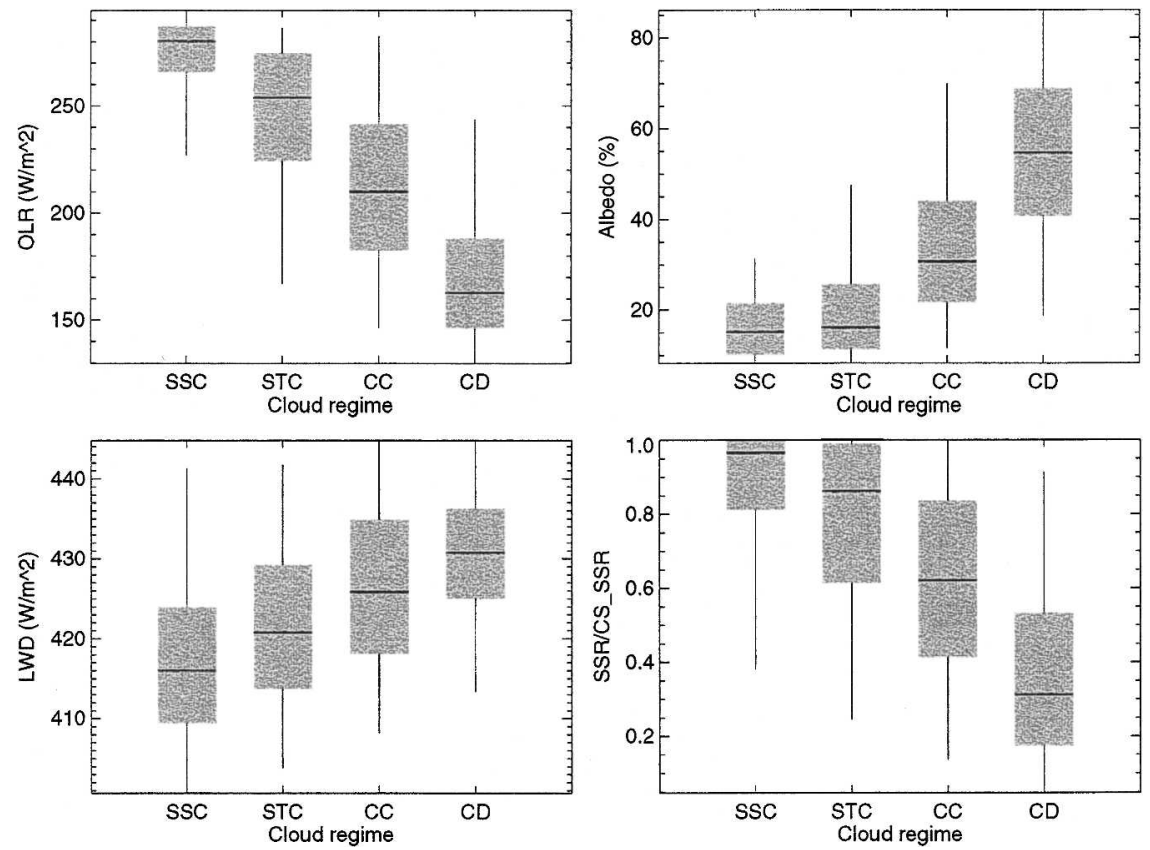

FIG. 3. Box whisker representation of the radiative characteristics measured at the Manus ARM site as a function of cloud regime (see text for details): (top left) OLR, (top right) visible TOA albedo, (bottom left) downward surface longwave radiation, and (bottom right) the ratio of downward surface solar radiation to clear-sky downward surface solar radiation.

regimes from most suppressed (SSC) to convectively most active (CD) is very prominent. It is also evident that both the $25 \%$ and $75 \%$ quartile values and the "extremes" (5\% and $95 \%$ percentiles) show signals in accordance with the median values. Just as the OLR decreases from SSC to CD, the albedo increases from values around $15 \%$ for SSC to about $55 \%$ for the CD regime. These results are consistent with the mean ISCCP CTP $-\tau$ histograms for the regimes shown in Fig. 1. The SSC regime at Manus has a low value of total cloud cover (0.36), and if any high-level clouds exist, they tend to be thin. This leads to both low albedo and high OLR values at the TOA. The STC cloud cover is significantly higher than that of SSC (0.78) but is dominated by relatively thin cirrus clouds. Hence the albedo increases slightly and the OLR decreases by moderate amounts in comparison to SSC. The CC regime exhibits higher total cloud cover (0.93), more clouds with low CTP, and optically thicker clouds than STC, explaining the further reduction in OLR and increase in albedo. While the cloud cover in the $\mathrm{CD}$ regime is not very different from that of CC (0.99), the clouds are optically much thicker, and there is a higher frequency of low CTP clouds. The CD regime therefore exhibits the lowest OLR and highest albedo of the four regimes.

The effect of the cloud regimes on solar radiation is confirmed at the surface (Fig. 3, bottom), where the normalized median solar radiation values drop from near 1 for the SSC regime to close to 0.3 for the CD regime. The downward longwave radiation in the Trop- ics is usually less affected by clouds because of the abundance of water vapor in the lower troposphere. Nevertheless, Fig. 3 indicates that the different cloud regimes are associated with different values of downward longwave radiation, with a small increase in the median value from about $415 \mathrm{~W} \mathrm{~m}^{-2}$ for the SSC to just above $430 \mathrm{~W} \mathrm{~m}^{-2}$ for $\mathrm{CD}$. As will be shown below, this is likely due to the fact that the thermodynamic conditions in each regime, in particular the water vapor content, are significantly different, but an increase in the area covered by cloud bases also contributes to this effect. Cloud-base heights of the lowest cloud layers do not vary significantly between regimes, making it unlikely that the location of cloud base is a major factor in the observed downward longwave radiation variations.

\section{b. Cloud characteristics}

In this section, vertical cloud structure information derived from a suite of active remote sensing instruments located at the Manus ARM site is used to describe the cloud fields in each of the regimes in more detail. Because of the capabilities of the active instruments in penetrating clouds, this analysis also serves to provide information about the cloud fields that is not available from passive remote sensors as flown on satellites. The dataset used in this part of the study is the Active Remotely Sensed Cloud Layers (ARSCL) data (Clothiaux et al. 2000). This dataset provides observations of the location in the vertical of cloud layers observed by a combination of a $35-\mathrm{GHz}$ cloud radar (Mo- 
ran et al. 1998; Clothiaux et al. 1999) and a micropulse lidar (Spinhirne 1993) in 10-s intervals. In particular, information on the location of cloud bases and tops in the column above is provided. Seventeen months of data from August 1999 to December 2000 are used here. As for radiation, periods of missing and erroneous data have been removed.

In a first analysis, the data are converted into hourly vertical profiles of cloud cover (or more correctly, hydrometeor cover, since the radar is sensitive to both cloud and precipitation size particles) in 100-m height intervals. These profiles are generated by simply counting the number of times within the hour in which a cloud is identified in every $100-\mathrm{m}$ bin and dividing by the total number of observations taken within the hour. As for the radiation parameters above, the cloud cover profiles are then time matched with the available cloud regime information and assigned to the regime occurring at a given time. Figure 4 summarizes the results. The SSC regime is characterized by a peak in low-level cloud cover and a second small maximum at around 13 $\mathrm{km}$. An even smaller peak around $16 \mathrm{~km}$, indicative of cirrus located very near the tropopause, is also evident. Low-level cloud cover in the STC regime is very similar to that in the SSC regime, but the coverage with cirrus both around $13 \mathrm{~km}$ and around $16 \mathrm{~km}$ has increased significantly. The latter is consistent with the ISCCP histograms shown in Fig. 1, which show a larger overall cloud cover dominated by thin cirrus in STC compared to SSC. However, it is interesting to note for this regime, and more so for the convectively active ones, that the low cloud cover as seen by the satellite is decreasing while the active remote sensors indicate an increase in low cloud cover. This is of course due to the passive character of the satellite measurements, which prevents penetration of optically thick clouds and leads to the inability of the satellite instruments to detect low-level clouds in the presence of upper-level clouds. The cloud regime separation advocated in this study shows some promise for a synergistic application of both data sources together, thereby extending the use of both beyond their own limitations. The two convective regimes (CC and CD) show the largest cloud covers; in particular, CD exhibits high cloud covers in the middle troposphere. It is important to recall that the cloud radar, which forms the most important contributor to the ARSCL dataset, is sensitive to precipitation-size particles. Hence the large cloud cover of the layers below about $5 \mathrm{~km}$ is likely at least partly due to precipitation. Another noteworthy feature of Fig. 4 is the apparent reduction of cloud-top height as the convective activity is increasing. This may, however, well be due to the attenuation of the radar signal, since the most convectively active periods are prone to producing the clouds/ precipitation layers of the largest vertical extent. Finally, it is worthwhile to draw the attention to a maximum in cloud cover near the $5-\mathrm{km}$ level, which is pronounced in all regimes but SSC. This feature draws

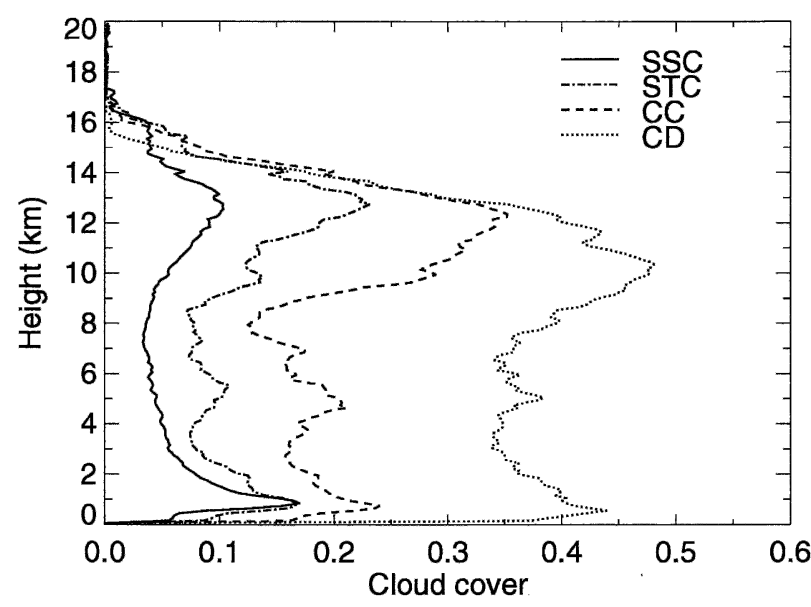

FIG. 4. Vertical profile of cloud cover derived from the ARSCL product as a function of cloud regime.

attention because of the recent interest in the presence and role of convective clouds with tops near the melting layer (mostly cumulus congestus; e.g., Johnson et al. 1999; Mapes 2000). A further investigation as to whether this maximum is indeed a reflection of such clouds will be carried out below.

Whilst certainly instructive, the use of average cloud cover profiles does not provide much information on the actual cloud macrostructure that is observed in the different regimes. To elucidate this structure in more detail, the ARSCL data are used to construct twodimensional histograms of the frequency of occurrence of clouds of a certain cloud-top height and cloud physical thickness, much in the spirit of the ISCCP CTP- $\tau$ histograms. These histograms are constructed hourly by assessing all cloud layers present in a 10-s interval for their thickness and top height and calculating the frequency of occurrence over the hour of the combination of height and thickness in 2-km intervals. As for the data above, for each hour that a cloud regime classification is available, the ARSCL histograms are then sorted by regime, and the resulting average histograms for each regime are displayed in Fig. 5.

Before interpreting the figure, it seems prudent to provide a short tutorial on the diagrams contained in it. Clouds are grouped by their physical thickness and cloud-top height, and, as in the ISCCP histograms, the shading corresponds to the frequency of occurrence of clouds with a certain combination of these two characteristics. Note that it is a reasonable assumption that the frequency of occurrence in time at a single site corresponds to spatial cloud cover when averaged over long enough periods. Since each of the histograms in Fig. 5 contains several hundred hours of data, this assumption holds for the results presented here. Analogous to the ISCCP histograms, certain types of clouds populate certain parts of the diagram. For instance, cirrus clouds, which are thin clouds with high tops, can be identified in the top left corner. Convective clouds will populate 

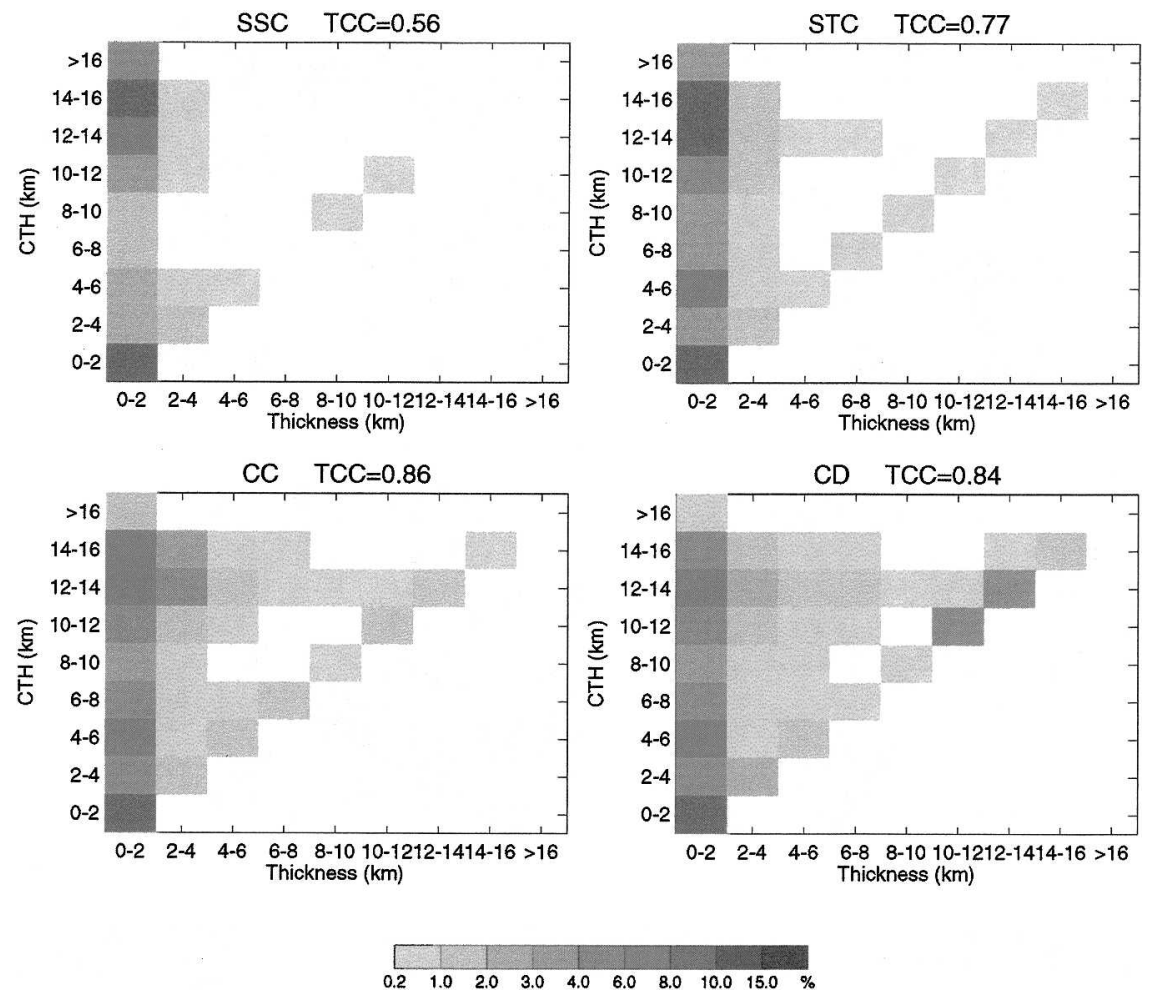

FIG. 5. Regime-averaged histograms of the frequency of occurrence of clouds of a given cloud-top height ( $y$ axis) and cloud thickness ( $x$ axis) derived from the ARSCL data at Manus. The regimes are (top left) SSC, (top right) STC, (bottom left) CC, and (bottom right) CD. Data for Aug 1999 to Dec 2000 has been used in this analysis.

the diagonal. This is so, since over the tropical ocean, convective cloud base is generally low (a few hundred meters), so that the cloud-top height of such clouds is very close to their physical thickness. Shallow convection then populates the bottom left part of the diagonal, while deep convective clouds appear in the top right part. The apparent dominance of thin clouds, indicated by the high frequency of occurrence in the first column of each diagram warrants some further explanation. It is caused by the fact that at any given time, several thin cloud layers can occur simultaneously, while this is impossible for thick cloud layers simply because of the limited vertical extent of the troposphere. Since each observed cloud layer is given equal weight in the histogram, many more thin than thick layers are identified. The high population of very thin cloud layers is consistent with the findings of previous studies (e.g., Warren et al. 1985; Sheu et al. 1997; Wang et al. 2000), who find high frequencies of occurrence of often overlapping thin cloud layers in tropical cloud fields, mainly because cirrus clouds often overlay boundary layer clouds. However, as will be discussed in more detail below, this study reveals an additional layer of frequent occurrence of thin clouds located in the middle troposphere.

With this information in mind, the results in Fig. 5 can now be evaluated. The SSC regime is characterized by a high frequency of occurrence of low thin clouds of less than 2-km depth (shallow cumulus) and thin high clouds (cirrus). There are some occurrences of convective clouds up to $4-6-\mathrm{km}$ depth, indicative of the presence of a congestus phase of convection. However, in the SSC regime, their frequency of occurrence tends to be low. In comparison to the SSC regime, the STC regime is marked both by an increase of the frequency of occurrence of thin cirrus and an increase in the thicker $(2-4 \mathrm{~km})$ high-top cloud classes. Also apparent in the figure is an increase of the presence of convective clouds of all depths up to $14-16 \mathrm{~km}$. This increase in convective activity continues when moving to the CC regime. This regime has the highest frequency of occurrence of medium-thick $(2-4 \mathrm{~km})$ cirrus clouds, consistent with the mean ISCCP histograms for this regime (cf. Fig. 1). There are also indications for the emergence of clouds of $6-8-\mathrm{km}$ thickness with high tops, indicative of cumulus anvils. The occurrence of these clouds is further increased in the CD regime, again consistent with the ISCCP classification of that regime. The CD regime is also marked with the largest occurrence of very deep clouds with their tops between 10 and $14 \mathrm{~km}$.

All of the above results are consistent with the expectations one would have if the cloud regime classification derived from ISCCP was meaningful. However, 
there are a number of cloud field characteristics that can be derived from the ARSCL product that are not detectable with the passive remote sensors used in ISCCP. First, low shallow clouds are the predominant tropical cloud type in all regimes. Convective clouds up to $4-6-\mathrm{km}$ depth are ubiquitous, with a dominance of those of less than 2-km depth. The frequency of the congestus-type clouds increases with deep convective activity, consistent with their role in supporting the development of deep convection postulated in many recent studies (Johnson et al. 1999; Mapes 2000). Equally ubiquitous in the Tropics are thin cirrus clouds. According to the analysis here, clouds of tops between 12-16 km and thicknesses of less than $2 \mathrm{~km}$ exhibit a very high frequency of occurrence. There is also an indication of a reasonable number of such clouds with tops above $16 \mathrm{~km}$, well within in the tropical tropopause layer (TTL). The frequency of occurrence of these higher cirrus clouds appears to be reduced in the two convectively active regimes as compared to the suppressed ones. As discussed above, this may, however, be an artifact of attenuation of the active remote sensors and should therefore not be overinterpreted.

There is one cloud type emerging in Fig. 5 that has received little attention in the literature so far. All regimes show a relative maximum in the occurrence of clouds with a top between $4-6 \mathrm{~km}$ and a thickness of less than $2 \mathrm{~km}$. The frequency of occurrence of these clouds increases with convective activity. While located at the top of the congestus layer, it is not obvious if these clouds constitute cumulus congestus "anvils" or whether they represent the shedding of cloud material from even deeper convective drafts as those encounter a layer of increased static stability near the melting level. One could also speculate that their increased occurrence in situations of deeper convection is related to a strengthening of that stable layer through the increased cooling induced by the melting precipitation from deep convection and, more importantly, the widespread anvil clouds associated with it. Since these thin midlevel cloud layers appear most strongly in periods of deep convection, their radiative effect may be overshadowed by those of the concurrent deeper anvil clouds and hence be of little importance. However, the presence of these clouds does indicate an increased relative humidity in the middle troposphere, and they may well play an important role in the maintenance of the moist, stable layer around $0^{\circ} \mathrm{C}$ beyond the lifetime of their parent convection.

\section{c. Thermodynamic characteristics}

After characterizing the radiative and cloud macrophysical properties associated with the major TWP cloud regimes, this section investigates some of their thermodynamic properties. For this purpose, two data sources at the ARM site are applied. First, retrievals of total column water vapor (TCWV) from a groundbased microwave radiometer (MWR) are used to char-

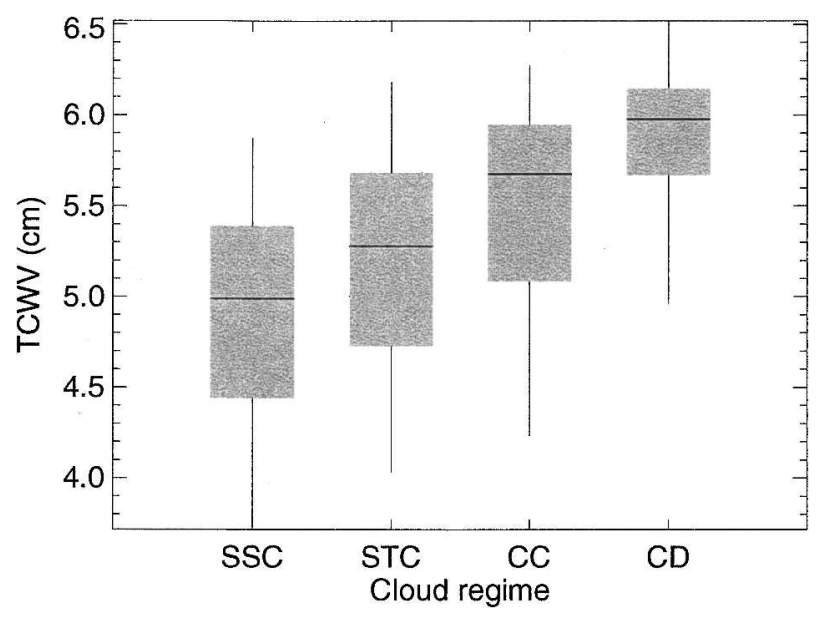

FIG. 6. Box whisker representation of the TCWV measured at the Manus ARM site as a function of cloud regime (see text for details).

acterize the overall water vapor content in each regime. Details of the TCWV retrievals can be found in Liljegren (1994). As for the radiation data above, the highfrequency $(20 \mathrm{~s})$ observations at the ARM site are averaged to hourly mean values. Special care had to be taken in the quality control of this data, since MWR measurements are susceptible to large errors in the presence of precipitation, a frequent occurrence at Manus. The instrument is equipped with a heaterblower unit in an attempt to keep the upward-looking instrument window clear of rainwater. However, in order to eliminate erroneous data as best as possible, all hours for which this unit was active for more than half the time have been eliminated. After assessing the vertically integrated mean water vapor structure, radiosonde data are used to assess the mean vertical thermodynamic profiles within each regime. Note that because of the availability of the ISCCP data during daytime only and the 12-hourly interval in radiosonde launches, only one sounding per day is available. This limited sampling is especially acute for the CD regime, which has the lowest frequency of occurrence. The radiosonde humidity data have been corrected in an ad hoc fashion by scaling the relative humidity at each level with a factor derived by comparing the TCWV of the sounding with that of the MWR retrievals to ensure a match of TCWV between the two instruments. This technique generally leads to a moistening of the radiosonde profiles. While certainly of importance for detailed quantitative studies of tropical water vapor, the results presented below and their (mostly qualitative) interpretation do not depend on the details of this correction.

Figure 6 shows a box whisker representation of the TCWV values within each regime, analogous to radiation in Fig. 3. The median value of TCWV increases with increased convective activity from values of $5 \mathrm{~cm}$ 
SSC

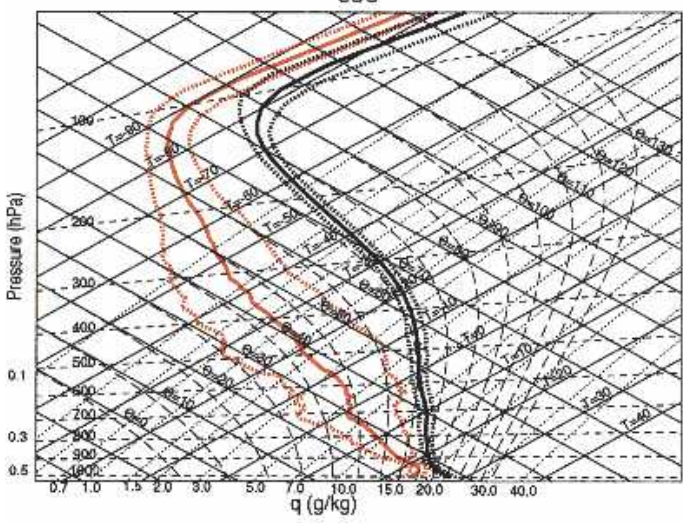

$\mathrm{CC}$

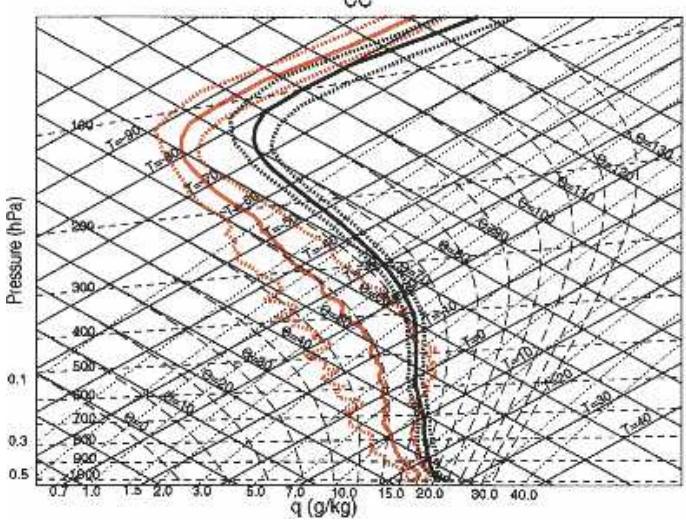

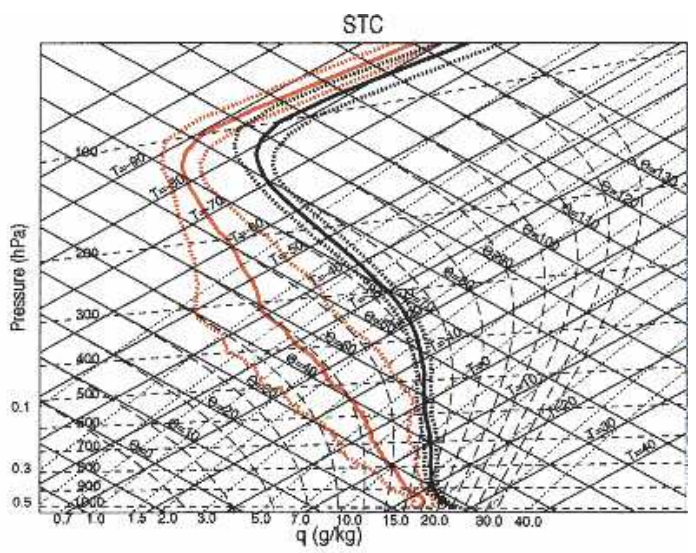

$C D$

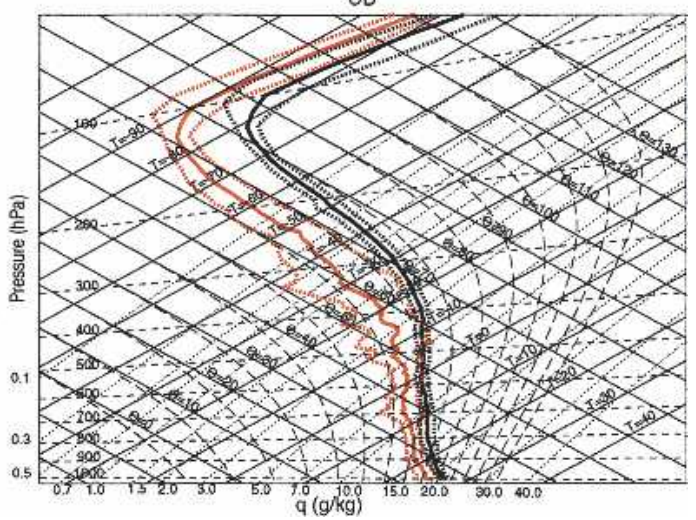

FIG. 7. Regime-averaged soundings for the (top left) SSC, (top right) STC, (bottom left) CC, and (bottom right) $\mathrm{CD}$ cloud regimes at the Manus ARM site. Solid lines represent mean temperature (black) and dewpoint temperature (red), respectively. Dotted lines indicate one standard deviation for all soundings within the regime.

for the $\mathrm{SSC}$ regime to about $6 \mathrm{~cm}$ in the $\mathrm{CD}$ regime. It is noteworthy, and will be shown in more detail below, that this increase is mainly due to increased middletropospheric humidity in the more convectively active regimes. While the absolute value of TCWV at any location in the Tropics may be dominated by low-level humidity, its variability is almost entirely due to midtropospheric variations in moisture. There is an apparent reduction in within-regime variability in the $\mathrm{CD}$ regime compared to the other three regimes. While this might be due to the upper bound of humidity at saturation, it may also be a reflection of the fact that this regime is poorly sampled in the MWR data as a result of the frequent presence of precipitation, and hence absence of reliable data, when this regime is present. It is impossible to distinguish the two effects with the available data.

Figure 7 shows a tephigram representation of the profiles of temperature and dewpoint temperature for each of the TWP regimes derived from radiosonde data. The thick solid lines indicate the regime mean profiles whilst the dotted lines on either side of the mean denote the standard deviation of all profiles within the regime. The most prominent feature in Fig. 7 is the gradual increase in midlevel humidity starting from a very dry state in the SSC regime to what is a "typical" tropical sounding in the presence of deep convection in the $C D$ regime. As already alluded to above, the low-level humidity is changing only marginally, and the biggest signal in the TCWV variance seen in Fig. 6 is likely due to variations in the levels between 900 and $500 \mathrm{hPa}$, since levels above that contribute little to the vertical integral as a result of their small absolute moisture contents. As is typical for the Tropics, the withinregime variability, indicated by the dotted lines, is small for temperature and substantial for dewpoint temperature. The fact that there is still substantial vertical noise, especially in the dewpoint profiles for the CC and CD regimes, suggests that despite the use of $2 \mathrm{yr}$ of data, sampling problems remain an issue. In spite of this, Fig. 7 does constitute a sufficiently accurate, if qualitative, picture of the thermodynamic conditions associated with each of the TWP cloud regimes.

Finally, how precipitation varies between the different cloud regimes is investigated. Hourly averages of precipitation from a single rain gauge that forms part of the ARM ARCS instrumentation is stratified by cloud regime much in the same way as the radiation measure- 
ments above. Given the large spatial variability of precipitation in the Tropics, results from a single gauge should be treated with caution, and only a qualitative interpretation will be attempted here. Table 1 shows the relative frequency of occurrence of precipitation within each cloud regime as well as the median of the distribution of the precipitation amounts when precipitation is present. The relative number of times that precipitation is registered by the gauge increases from about $20 \%$ in the suppressed regimes to $56 \%$ in the CD regime. This is indicative of the larger likelihood of the existence of precipitating clouds in the convectively active regimes as well as the larger within-regime coverage with such clouds, in particular in the CD regime. The amounts of precipitation when present are very small for the SSC regime. In fact it is only the CD regime that produces significant amounts of precipitation usually associated with convective systems in the Tropics. Keeping the above-mentioned caveats of using a single rain gauge in mind, the only conclusion drawn here is that the precipitation information available, like all the other data sources used so far, is consistent with the cloud regime definitions proposed in this study.

\section{Discussion}

The main characteristics of the four major TWP cloud regimes identified by JT03 have been described using a variety of data sources at the ARM site on Manus Island. The intuitive interpretation of the regimes as convectively active and suppressed modes with differing cloud configurations attempted in JT03 based on the ISCCP results alone has been confirmed by the use of these data sources, such as surface and TOA radiative fluxes, cloud macrophysical information, and thermodynamic profile information.

One of the main questions at the outset of this study was whether it is possible to identify recurring cloud regimes in the TWP from ISCCP data such that the resulting regimes have physical meaning beyond the information that was used in their definition. Answering that question is somewhat hampered by the fact that while all the data used here to describe the properties of the cloud regimes are technically independent of the ISCCP data, some of the parameters used, in particular those related to surface and TOA radiation, are of course strongly linked to the ISCCP properties that form the basis of the regime definition. Nevertheless, the fact that for each of the many observation types used here, distinct regime properties can be identified lends some support to the hypothesis that the TWP cloud regimes identified in JT03 do indeed represent important modes of tropical cloudiness. A caveat in that context results from the use the ISCCP CTP $-\tau$ histograms for the cloud regime definition, since they only exist during daytime. It is well known that convective systems over the tropical oceans exhibit a diurnal cycle (e.g., Sui et al. 1997), so that sampling the cloud
TABLE 1. Relative frequency of occurrence (RFO) and median amount when present (AP) for precipitation registered at a single gauge on Manus Island within each of the TWP cloud regimes.

\begin{tabular}{lcccc}
\hline \hline & SSC & STC & CC & CD \\
\hline RFO & 0.19 & 0.22 & 0.31 & 0.56 \\
AP $\left(\mathrm{mm} \mathrm{h}^{-1}\right)$ & 0.01 & 0.08 & 0.08 & 0.40
\end{tabular}

regimes during daytime only somewhat limits the conclusions that can be drawn for their more general behavior. So far, no solution to this caveat exists. However, the use of data away from major land masses in this study should minimize the diurnal cycle effects, since the amplitude of the diurnal cycle over the ocean tends to be small. Investigations are currently underway into the use for regime identification of data sources that are independent of solar radiation information or the extension of the existing regime information to nighttime by using the ISCCP infrared information only.

Further trust in the physical meaning of the regimes could be gained if a link to the dynamic behavior of the atmosphere could also be shown. Unfortunately, no reliable measurements for the key dynamical characteristics of the tropical atmosphere, namely convergence and divergence as a function of altitude or, equivalently, profiles of vertical velocity, do exist. In an attempt to at least qualitatively link the cloud regimes to tropical dynamics, vertical pressure velocity $(\omega)$ profiles derived from four different NWP analysis systems, are stratified into the observed cloud regimes for the years 1999-2000. The NWP systems used are the European Centre for Medium-Range Forecasts (ECMWF) operational analysis, the most recent ECMWF reanalysis [the 40-yr ECMWF Re-Analysis (ERA-40)], the National Centers for Environmental Prediction (NCEP) reanalysis, and the Australian Bureau of Meteorology operational analysis [Global Assimilation and Prediction System (GASP)]. Six-hourly analyses from all systems are interpolated to a $2.5^{\circ} \times 2.5^{\circ}$ regular grid. Vertical velocity profiles for the grid area containing Manus are time-matched with the cloud regime information, and the regime-averaged profiles for all four analyses are displayed in Fig. 8.

The first and most obvious feature in Fig. 8 is that despite the long averaging period of $2 \mathrm{yr}$, even the mean profiles of vertical velocity between the four NWP analyses show very little agreement. While perhaps not entirely unexpected, this result is somewhat discouraging for the use of these products in describing even the mean dynamic conditions in the Tropics, at least locally. Despite the disagreement in the mean, though, there is remarkable agreement between all analyses in the relative change in the vertical velocity profiles with cloud regime. In all analyses, the $\mathrm{CD}$ regime shows the strongest ascent, while the most suppressed regime (SSC) shows the largest positive anomaly in $\omega$. However, only ECMWF and ERA-40 show mean subsi- 
Average ecmwf vertical velocity

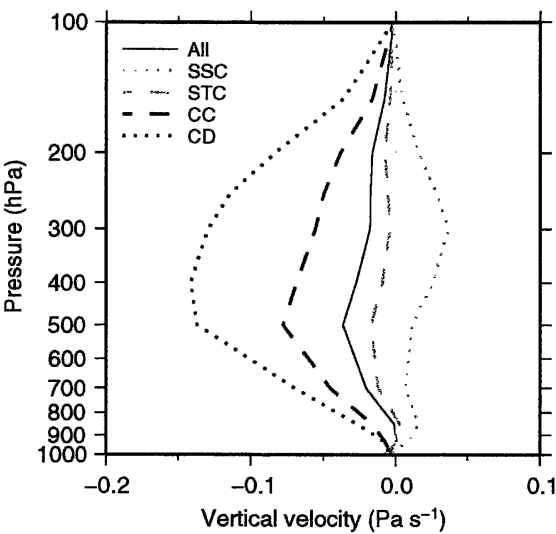

Average ncep vertical velocity

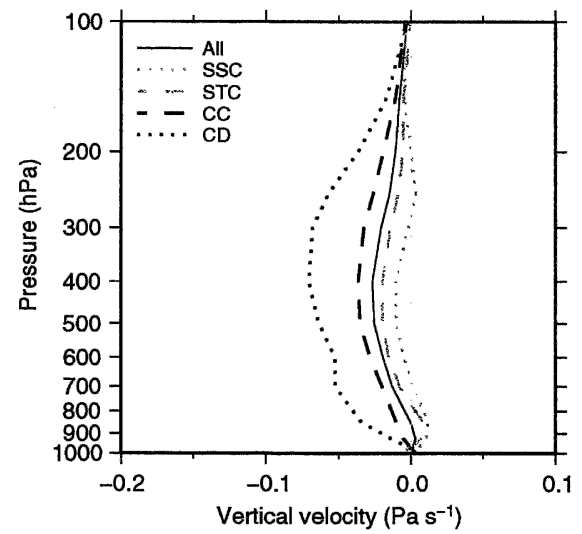

Average era40 vertical velocity

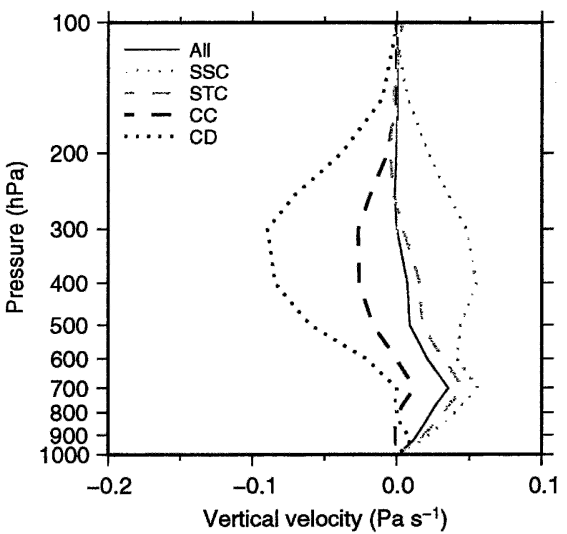

Average gasp vertical velocity

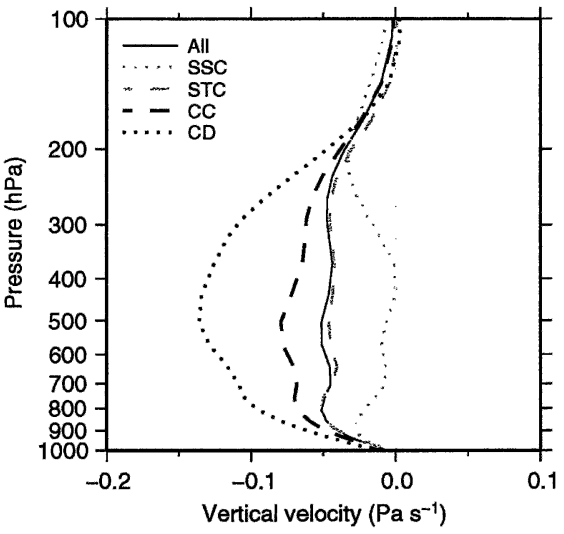

FIG. 8. Regime-averaged vertical velocity profiles soundings for (top left) the operational ECMWF analysis, (top right) ERA-40, (bottom left) the NCEP reanalysis, and (bottom right) the Australian Bureau of Meteorology operational analysis.

dence in that regime. Because of the large quantitative discrepancies between the NWP analyses, it remains difficult to argue that these figures provide conclusive proof that the ISCCP cloud regimes are indicative for tropical modes. However, the consistency of the results with those in the previous section at least strengthens that hypothesis.

Assuming that this study has identified and described major modes of cloudiness in the TWP, this still warrants the question of the usefulness of that information. There are a variety of potential applications for the ideas and cloud regime information presented here.

An obvious application, and one of the main driving factors for carrying out this study, is the potential use of cloud regime information for the identification of regime-dependent errors in numerical models. This is of quite some significance, since in particular the GCM community has been searching for means of analyzing models such that information not only on the existence of errors, but insight into their cause, is revealed. Analyzing model errors as a function of regime divides the overall model error into "local" within-regime errors, which the parameterization community can address in case studies, and errors in the frequency of occurrence of the individual regimes, which may or may not have local causes. The method will also help to reveal the ever-present compensating errors in models, by potentially exposing an apparent small error as a combination of large but canceling within-regime and/or occurrence errors.

Another potential application of the regime thinking is in the transfer of knowledge gained through measurements at an individual site to other locations or even larger-scale areas. In the case presented here, for instance, the within-regime vertical profiles of cloud (hydrometeor) cover are known at the Manus site. This kind of information is currently unavailable even on a regional scale, since it relies on the presence of active remote sensing instruments, which are only sparsely deployed over the globe. It is an interesting thought to use the globally available cloud regime information to extrapolate point measurements of, say, cloud cover profiles to other regions by assuming that they are a linear combination of within-regime cloud cover and fre- 
quency of occurrence of a regime. The potential of this idea will be explored in future work.

Cloud feedbacks have been in the limelight of climate change discussions for many years. While this study was able to describe the radiative and cloud properties of the cloud regimes, their relation to dynamical features of the tropical atmosphere, such as tropical waves, remains to be explored. However, the success in discriminating the regime characteristics in this study provides encouragement for the use of the cloud regime framework to better our understanding of cloud feedbacks in the atmosphere. This could be achieved by using the quantitative information on both regime occurrence and characteristics provided here to separate cloud effects and their variability into within-regime changes and changes of the frequency of occurrence of the regimes. A study of cloud effects relating to ENSO variability using this framework is currently underway.

Field experiments and case study selection for modeling studies are other potential benefactors of cloud regime thinking. As was demonstrated for one of the ARM sites in Fig. 1, the separation of the mean cloud fields into regimes can help put results of a single site and/or a distinct period of measurements into context. Furthermore, typical cases observed during an experiment can be identified and used for further analysis by both the observational and modeling community.

Last but not least, this study has demonstrated the enormous usefulness of long-term observations as carried out by ARM and other field programs as well as long-term satellite datasets. These observations provide a comprehensive "archive" of many cases and when put into context through techniques like that proposed here can contribute to our understanding of cloud and radiative processes far beyond the sites at which they were taken.

\section{Conclusions}

The multitude of cloud fields encountered in the TWP has been shown to be composed of four major regimes at a scale of about $300 \mathrm{~km} \times 300 \mathrm{~km}$. ARM data have been used to characterize the radiative, cloud, and thermodynamic properties of the regimes. The four regimes are as follows:

1) SSC - a suppressed (in the deep convective sense) cloud regime dominated by low-top clouds of low to medium $\tau$, most likely shallow convective clouds. This regime is characterized by high values of OLR, low albedo, and small cloud effects on both solar and longwave radiation. Furthermore, the regime is shown to exist in situations of low mid- and uppertropospheric humidity. This regime is the most frequent of those identified in the TWP.

2) STC-a suppressed cloud regime, dominated by thin cirrus clouds. This regime, while still existing in relatively low-tropospheric humidity conditions, ex- hibits a larger overall cloud cover and lower OLR but only slightly higher albedo than SSC.

3) $\mathrm{CC}$-a regime with a small coverage of what are likely deep convective clouds and a large coverage of high-top medium- $\tau$ cirrus. This regime shows a significant cloud effect both in longwave and shortwave radiation. The median OLR of the regime is around $210 \mathrm{~W} \mathrm{~m}^{-2}$, roughly $50 \mathrm{~W} \mathrm{~m}^{-2}$ lower than in the suppressed regimes; the albedo is around $30 \%$. The mean thermodynamic properties of this regime approach that of deep convection with TCWV values of more than $5.5 \mathrm{~cm}$

4) CD—a regime dominated by a large coverage with high-top clouds of substantial optical thickness. Thermodynamically, this regime is the closest to what is termed a classic tropical convection profile. Radiatively, this is the regime with the largest effect, but it has the lowest frequency of occurrence in both the TWP and at the Manus ARM site. Its median OLR is below $170 \mathrm{~W} \mathrm{~m}^{-2}$, the TOA albedo exceeds $50 \%$ on average, and the TCWV is close to $6 \mathrm{~cm}$.

It has been demonstrated that sorting the wide variety of cloud fields occurring in the TWP into major regimes facilitates the use of observations at a single location to describe the main characteristics of the regimes themselves. This is a nontrivial conclusion since it is much easier to collect complex and comprehensive sets of observations, such as those at the ARM CART sites, at a single location than over a large area. The synergy of these complex observations with the "simpler" ones provided in the ISCCP dataset provides a potentially powerful tool in understanding cloud features over a wider area from those observed at a location. Quite how well this extrapolation can be made to work remains an area of active research. In this context, it has been shown that long datasets at a single location, such as those provided by ARM, provide an invaluable source of data since they make it possible to build large enough samples of cases for each regime.

Several examples for applications of the cloud regime identification have been highlighted. Those range from model evaluation, to understanding cloud effects at single locations as within-regime effects combined with a frequency of occurrence of the regime, to extrapolating single-site results to larger areas, to cloud feedback studies. It would be foolish to claim that the approach described in this paper will by itself solve these problems, but enough evidence has been provided that it constitutes a new and useful addition to the arsenal of tools already in existence.

Acknowledgments. We thank Chuck Long for providing the clear-sky radiation data at Manus. Pat Minnis is acknowledged for providing the satellite retrievals of OLR and albedo. William B. Rossow and Harry Hendon provided us with comments on an early version of the manuscript. We thank Robert Pincus for several 
heated discussions on this work. Three anonymous reviewers are acknowledged for their very helpful comments. Their collective insight has led to significant improvements of the manuscript. We are grateful for support from the U.S. Department of Energy under grants DE-FG02-03ER63533 and LANL-23662-001-013T as part of the Atmospheric Radiation Measurement Program.

\section{REFERENCES}

Ackerman, T. P., and G. M. Stokes, 2003: The Atmospheric Radiation Measurement Program. Phys. Today, 56, 38-44.

Anderberg, M. R., 1973: Cluster Analysis for Applications. Academic Press, $359 \mathrm{pp}$

Bony, S., K.-M. Lau, and Y. Sud, 1997: Sea surface temperature and large-scale circulation influences on tropical greenhouse effect and cloud radiative forcing. J. Climate, 10, 2055-2077.

Browning, K. A., 1993: The GEWEX Cloud System Study (GCSS). Bull. Amer. Meteor. Soc., 74, 387-399.

Clothiaux, E. E., and Coauthors, 1999: The Atmospheric Radiation Measurement Program cloud radars: Operational modes. J. Atmos. Oceanic Technol., 16, 819-827.

, T. P. Ackerman, G. G. Mace, K. P. Moran, R. T. Marchand, M. A. Miller, and B. E. Martner, 2000: Objective determination of cloud heights and radar reflectivities using a combination of active remote sensors at the ARM CART sites. $J$. Appl. Meteor., 39, 645-665.

Houghton, J. T., Y. Ding, D. J. Griggs, M. Noguer, P. J. van der Linden, X. Dai, K. Maskell, and C. A. Johnson, Eds., 2001: Climate Change 2001: The Scientific Basis. Cambridge University Press, $881 \mathrm{pp}$.

Jakob, C., 2003: An improved strategy for the evaluation of cloud parameterizations in GCMs. Bull. Amer. Meteor. Soc., 84, 1387-1401.

— regimes in the Tropical Western Pacific. Geophys. Res. Lett., 30, 2082, doi:10.1029/2003GL018367.

Johnson, R. H., T. M. Rickenbach, S. A. Rutledge, P. E. Ciesielski, and W. H. Schubert, 1999: Trimodal characteristics of tropical convection. J. Climate, 12, 2397-2418.

Lau, N.-C., and M. W. Crane, 1995: A satellite view of the synoptic-scale organization of cloud properties in midlatitude and tropical circulation systems. Mon. Wea. Rev., 123, 19842006.

Liljegren, J. C., 1994: Two-channel microwave radiometer for observations of total column precipitable water vapor and cloud liquid water path. Preprints, Fifth Symp. on Global Change Studies, Nashville, TN, Amer. Meteor. Soc., 262-269.

Long, C. N., and T. P. Ackerman, 2000: Identification of clear skies from broadband pyranometer measurements and calculation of downwelling shortwave cloud effects. J. Geophys. Res., 105, 15 609-15 626.

— K. L. Gaustad, 2004: The shortwave (SW) clear-sky detection and fitting algorithm: Algorithm operational details and explanations. ARM Value-Added Procedure Rep. TR-004.1, $24 \mathrm{pp}$. [Available online at http://www.arm.gov/publications/ techreports.stm.]

Mapes, B. E., 2000: Convective inhibition, subgrid-scale triggering energy, and stratiform instability in a toy tropical wave model. J. Atmos. Sci., 57, 1515-1535.

Mather, J. H., T. P. Ackerman, W. E. Clements, F. J. Barnes, M. D. Ivey, L. D. Hatfield, and R. M. Reynolds, 1998: An atmospheric radiation and cloud station in the tropical western Pacific. Bull. Amer. Meteor. Soc., 79, 627-642.
Minnis, P., W. L. Smith Jr., D. P. Garber, J. K. Ayers, and D. R. Doelling, 1995: Cloud properties derived from GOES-7 for the spring 1994 ARM intensive observing period using version 1.0.0 of the ARM satellite data analysis program. NASA Reference Publication 1366, $59 \mathrm{pp}$.

Moran, K. P., B. E. Martner, M. J. Post, R. A. Kropfli, D. C. Welsh, and K. B. Widener, 1998: An unattended cloudprofiling radar for use in climate research. Bull. Amer. Meteor. Soc., 79, 443-455.

Nordeen, M. L., D. R. Doelling, M. M. Khaiyer, A. D. Rapp, P. Minnis, and L. Nguyen, 2001: GMS-5 satellite-derived cloud properties over the tropical western Pacific. Proc. 11th ARM Science Team Meeting, Atlanta, GA, U.S. Department of Energy Atmospheric Radiation Program. [Available online at http://www.arm.gov/publications/proceedings/conf11/ index.stm.]

Norris, J., and C. Weaver, 2001: Improved techniques for evaluating GCM cloudiness applied to the NCAR CCM3. J. Climate, 14, 2540-2550.

Randall, D. A., K.-M. Xu, R. J. C. Somerville, and S. Iacobellis, 1996: Single-column models and cloud ensemble models as links between observations and climate models. J. Climate, 9 , $1683-1697$.

- and Coauthors, 2003: Confronting models with data-The GEWEX Cloud Systems Study. Bull. Amer. Meteor. Soc., 84, 455-469.

Rossow, W. B., and R. A. Schiffer, 1999: Advances in understanding clouds from ISCCP. Bull. Amer. Meteor. Soc., 80, 22612287.

Schiffer, R. A., and W. B. Rossow, 1983: The International Satellite Cloud Climatology Project (ISCCP): The first project of the World Climate Research Program. Bull. Amer. Meteor. Soc., 64, 779-784.

Sheu, R., J. Curry, and G. Liu, 1997: Vertical stratification of tropical cloud properties as determined from satellite. J. Geophys. Res., 102, 4231-4246.

Spinhirne, J. D., 1993: Micro pulse lidar. IEEE Trans. Geosci. Remote Sens., 31, 48-55.

Stephens, G. L., 2003: The useful pursuit of shadows. Amer. Sci., 91, 442-449.

Stokes, G. M., and S. E. Schwartz, 1994: The Atmospheric Radiation Measurement (ARM) Program: Programmatic background and design of the cloud and radiation test bed. Bull. Amer. Meteor. Soc., 75, 1201-1221.

Sui, C.-H., K.-M. Lau, Y. N. Takayabu, and D. A. Short, 1997: Diurnal variations in tropical oceanic cumulus convection during TOGA COARE. J. Atmos. Sci., 54, 639-655.

Tselioudis, G., and C. Jakob, 2002: Evaluation of midlatitude cloud properties in a weather and a climate model: Dependence on dynamic regime and spatial resolution. J. Geophys. Res., 107, 4781, doi:10.1029/2002JD002259.

- Y. Yhang, and W. B. Rossow, 2000: Cloud and radiation variations associated with northern midlatitude low and high sea level pressure regimes. J. Climate, 13, 312-327.

Wang, J., W. B. Rossow, and Y. Zhang, 2000: Cloud vertical structure and its variations from a 20-yr global rawinsonde dataset. J. Climate, 13, 3041-3056.

Warren, S. G., C. J. Hahn, and J. London, 1985: Simultaneous occurrence of different cloud types. J. Appl. Meteor., 24, 658667.

Webb, M., C. Senior, S. Bony, and J.-J. Morcrette, 2001: Combining ERBE and ISCCP data to assess clouds in the Hadley Centre, ECMWF and LMD atmospheric climate models. Climate Dyn., 17, 905-922.

Williams, K. D., M. A. Ringer, and C. A. Senior, 2003: Evaluating the cloud response to climate change and current climate variability. Climate Dyn., 20, 705-721. 